\title{
Partial Quark-Lepton Universality and Neutrino CP Violation
}

\author{
Jiajun Liao, ${ }^{1,2}$ D. Marfatia, ${ }^{1}$ and K. Whisnant ${ }^{2}$ \\ ${ }^{1}$ Department of Physics and Astronomy, University of Hawaii at Manoa, Honolulu, HI 96822, USA \\ ${ }^{2}$ Department of Physics and Astronomy, Iowa State University, Ames, IA 50011, USA
}

Correspondence should be addressed to Jiajun Liao; liaoj@hawaii.edu

Received 1 June 2015; Accepted 29 July 2015

Academic Editor: Vincenzo Flaminio

Copyright (C) 2015 Jiajun Liao et al. This is an open access article distributed under the Creative Commons Attribution License, which permits unrestricted use, distribution, and reproduction in any medium, provided the original work is properly cited. The publication of this article was funded by SCOAP ${ }^{3}$.

We study a model with partial quark-lepton universality that can naturally arise in grand unified theories. We find that constraints on the model can be reduced to a single condition on the Dirac CP phase $\delta$ in the neutrino sector. Using our current knowledge of the CKM and PMNS mixing matrices, we predict $-32.4^{\circ} \leq \delta \leq 32.0^{\circ}$ at $2 \sigma$.

\section{Introduction}

Our understanding of neutrinos has progressed steadily in the last two decades. After the observation of nonzero $\theta_{13}$ by the Daya Bay [1, 2], RENO [3], and Double Chooz [4] experiments, we now know the three mixing angles $\theta_{12}$, $\theta_{23}$, and $\theta_{13}$ and the two mass squared differences to good precision. For the normal hierarchy, the current $2 \sigma$ ranges of the three mixing angles from a global three-neutrino oscillation analysis are [5]

$$
\begin{aligned}
& \theta_{12}=33.7_{-2.1}^{+2.1}\left(^{\circ}\right), \\
& \theta_{23}=41.4_{-2.6}^{+6.6}\left(^{\circ}\right), \\
& \theta_{13}=8.80_{-0.77}^{+0.73}\left(^{\circ}\right) .
\end{aligned}
$$

The focus of next generation neutrino oscillation experiments is shifted to the Dirac CP phase $\delta$ and the neutrino mass hierarchy. Predictions of many theoretical models designed to explain the observed mixing patterns await verification. Among these models, quark-lepton universality (QLU) [6] is well motivated. It is based on simple relations in grand unified theories (GUT) and connects the mixing matrices of quarks and leptons. Exact quark-lepton universality leads to a symmetric PMNS mixing matrix. However, using the current $3 \sigma$ ranges of the oscillation parameters [5], we find the moduli of the neutrino mixing matrix elements are

$$
\begin{aligned}
& \left|V_{\mathrm{PMNS}}\right| \\
& \quad=\left(\begin{array}{lll}
0.789-0.853 & 0.501-0.594 & 0.133-0.172 \\
0.195-0.556 & 0.410-0.733 & 0.602-0.784 \\
0.196-0.557 & 0.411-0.733 & 0.602-0.784
\end{array}\right) .
\end{aligned}
$$

We see that the exactly symmetric PMNS mixing matrix is disfavored by the current data. This aspect of the PMNS matrix with $V_{\mathrm{PMNS}}=V_{\mathrm{PMNS}}^{T}$ or $V_{\mathrm{PMNS}}=V_{\mathrm{PMNS}}^{\dagger}$ has been studied in [7-9].

In this paper, we discuss partial quark-lepton universality [6], which does not require the unitary matrices that diagonalize the upper and lower components of the weak doublets to be the same. We find that partial QLU fits the current data very well and we can make a prediction for the unknown Dirac CP phase.

In Section 2, we review partial quark-lepton universality and discuss renormalization group effects on the model. In Section 3, we discuss the phenomenological results of this model and predict the Dirac CP phase. We conclude in Section 4. 


\section{Partial Quark-Lepton Universality}

Partial quark-lepton universality can be derived from some simple relations in grand unified theories [6]. We start with the $\mathrm{SU}(5)$ relation

$$
M_{l}=M_{d}^{T}
$$

obtainable in lopsided models [10], and

$$
M_{u}=M_{u}^{T}
$$

where $M_{l}, M_{u}$, and $M_{d}$ are the mass matrices of the chargedleptons, up-type quarks, and down-type quarks, respectively. If we assume $M_{d}$ is Hermitian, which can be achieved by imposing left-right symmetry $[6]^{1}$, then from (3) we find that both the down-type quarks and charged-leptons can be diagonalized by a unitary matrix $V$

$$
\begin{aligned}
V^{\dagger} M_{d} V & =D_{d}, \\
V^{T} M_{l} V^{*} & =D_{l} .
\end{aligned}
$$

Also, from (4), we know that the up-type quarks can be diagonalized by a unitary matrix $V^{\prime}$

$$
V^{\prime \dagger} M_{u} V^{\prime *}=D_{u}
$$

If the Dirac neutrino matrix $M_{\nu D}$ and the right-handed Majorana neutrino mass matrix $M_{R}$ are also diagonalized by $V^{\prime}$ (as in some $\mathrm{SO}(10)$ models [6]),

$$
\begin{aligned}
V^{\prime \dagger} M_{\nu D} V^{\prime *} & =D_{\nu D}, \\
V^{\prime \dagger} M_{R} V^{\prime *} & =D_{R},
\end{aligned}
$$

Then, below the seesaw scale, the light neutrino mass matrix, $M_{v}=-M_{v D} M_{R}^{-1} M_{v D}^{T}$, is diagonalized by $V^{\prime}$ as well. Consider

$$
V^{\prime \dagger} M_{\nu} V^{\prime *}=D_{\nu}
$$

From (5), (6), and (8), we can find that the observable mixing matrices are related to

$$
\begin{gathered}
V_{\mathrm{CKM}}=V^{\prime \dagger} V, \\
V_{\mathrm{PMNS}}=V^{T} V^{\prime} .
\end{gathered}
$$

Note that, for exact quark-lepton universality, we must have $V^{\prime}=V$, which indicates that $V_{\mathrm{CKM}}=I$ and the $V_{\mathrm{PMNS}}$ mixing matrix is symmetric. This is disfavored by the current data. In the next section, we show that partial quark-lepton universality is still allowed by current data. A caveat to partial QLU is that small perturbations to the leading order relations of (9) and (10) are needed to reproduce the measured fermion masses. In [6], it was shown that, with a specific form for the perturbations, the measured fermion masses can be obtained while keeping the mixing matrices unchanged. Consequently, we focus on the connection between the mixing matrices of quarks and leptons. $^{2}$

The current data that determine the CKM and PMNS mixing matrices are measured at low energies, while the quark-lepton universality relations are realized at the grand unification scale. In order to use the current data to analyze the model, we must consider renormalization group (RG) effects. For the CKM matrix, the RG effects are very small; that is, the next order relative corrections to the CKM matrix are of the order $\lambda^{5}[11,12]$, where $\lambda=0.225$. The RG effects in the neutrino sector are strongly dependent on the mass spectrum of the light neutrinos. For the inverted and quasidegenerate mass hierarchies, the effects can be large [13-16]. However, with quark-lepton universality it is more natural to assume that the light neutrinos are very hierarchical with the normal mass spectrum. In this case, RG effects on the three angles are very small $[17,18]$; for example, $\delta \theta_{23} \sim 0.6^{\circ}$, $\delta \theta_{13} \sim 0.2^{\circ}$ and $\delta \theta_{12} \sim 0.8^{\circ}$ in the MSSM with $\tan \beta=$ 20 if the lightest neutrino mass is $0.01 \mathrm{eV}$. Since the current uncertainties in the three angles are larger than the RG effects, we neglect the RG effects in our analysis.

\section{Phenomenology}

In this section, we introduce a simple approach based on the properties of unitary matrices to reduce the constraints on the model to a single condition, which allows us to easily constrain the Dirac CP phase.

Partial QLU predicts the two observable mixing matrices to have the form of (9) and (10), which can be rewritten as

$$
\begin{aligned}
& V_{\mathrm{PMNS}} V_{\mathrm{CKM}}=V^{T} V, \\
& V_{\mathrm{CKM}}^{*} V_{\mathrm{PMNS}}=V^{\prime T} V^{\prime} .
\end{aligned}
$$

Hence, in order for the model to work, both $V_{\mathrm{PMNS}} V_{\mathrm{CKM}}$ and $V_{\mathrm{CKM}}^{*} V_{\mathrm{PMNS}}$ should be symmetric. However, the two constraints are not independent. Since (9) implies $V^{\prime}=$ $V V_{\text {CKM }}^{\dagger}$, (11) follows from (12).

Solutions for $V$ and $V^{\prime}$ will always exist because if $V_{\mathrm{CKM}}^{*} V_{\mathrm{PMNS}}$ is symmetric, then it can be diagonalized by a unitary matrix $U_{s}$; that is, $U_{s}^{T} V_{\mathrm{CKM}}^{*} V_{\mathrm{PMNS}} U_{s}=D$, where $D$ is diagonal. This means that we can find the solution, $V^{\prime}=$ $\sqrt{D} U_{s}^{\dagger}$. Once $V^{\prime}$ is known, the solution for $V$ can be obtained from (9). Although solutions for $V$ and $V^{\prime}$ exist, they are not unique. We can always insert a combination of a real rotation matrix $R^{T} R$ into the middle of the right-handed side of (11) or (12). And since $R^{T} R=I$, the equation will not change. This can also be seen from (9) and (10). For any real rotation matrix $R, R V$ and $R V^{\prime}$ are also unitary; hence if we let $V \rightarrow R V$ and $V^{\prime} \rightarrow R V^{\prime}$, the two observable mixing matrices will remain the same.

Now, if we define

$$
U=V_{\mathrm{CKM}}^{*} V_{\mathrm{PMNS}} \text {, }
$$

then the only constraint from the model is that $U$ is symmetric. Since both $V_{\mathrm{CKM}}$ and $V_{\mathrm{PMNS}}$ are unitary matrices, $U$ is also unitary. For a $3 \times 3$ unitary matrix, it can be shown that $U$ being symmetric is equivalent to the moduli of $U$ being symmetric under phase redefinition [19]. This constraint still imposes three conditions: $\left|U_{12}\right|=\left|U_{21}\right|,\left|U_{13}\right|=\left|U_{31}\right|$, and $\left|U_{23}\right|=\left|U_{32}\right|$. However, the conditions are not independent. 
Since $U$ is unitary, $\left|U_{11}\right|^{2}+\left|U_{12}\right|^{2}+\left|U_{13}\right|^{2}=\left|U_{11}\right|^{2}+\left|U_{21}\right|^{2}+$ $\left|U_{31}\right|^{2}$. Hence, $\left|U_{12}\right|=\left|U_{21}\right|$ indicates $\left|U_{13}\right|=\left|U_{31}\right|$ and vice versa. Similarly, $\left|U_{23}\right|=\left|U_{32}\right|$ is equivalent to $\left|U_{13}\right|=$ $\left|U_{31}\right|$. Therefore, there is only one independent condition that constrains the model. Here, we choose it to be $\left|U_{13}\right|=\left|U_{31}\right|$.

The CKM matrix can be written in terms of the Wolfenstein parameters [20] as follows:

$$
\begin{aligned}
V_{\mathrm{CKM}}= & {\left[\begin{array}{ccc}
1-\frac{\lambda^{2}}{2} & \lambda & A \lambda^{3}(\rho-i \eta) \\
-\lambda & 1-\frac{\lambda^{2}}{2} & A \lambda^{2} \\
A \lambda^{3}(1-\rho-i \eta) & -A \lambda^{2} & 1
\end{array}\right] } \\
& +\mathcal{O}\left(\lambda^{4}\right),
\end{aligned}
$$

and the PMNS matrix can be written in the standard form, which is

$$
\begin{aligned}
& V_{\text {PMNS }} \\
& =\left[\begin{array}{ccc}
c_{13} c_{12} & c_{13} s_{12} & s_{13} e^{-i \delta} \\
-s_{12} c_{23}-c_{12} s_{23} s_{13} e^{i \delta} & c_{12} c_{23}-s_{12} s_{23} s_{13} e^{i \delta} & s_{23} c_{13} \\
s_{12} s_{23}-c_{12} c_{23} s_{13} e^{i \delta} & -c_{12} s_{23}-s_{12} c_{23} s_{13} e^{i \delta} & c_{23} c_{13}
\end{array}\right],
\end{aligned}
$$

where $c_{i j}, s_{i j}$ denote $\cos \theta_{i j}$ and $\sin \theta_{i j}$, respectively, and Majorana phases are not included. From (13), we see that the condition $\left|U_{13}\right|=\left|U_{31}\right|$ becomes

$$
\begin{aligned}
& \left|\left(1-\frac{\lambda^{2}}{2}\right) s_{13} e^{-i \delta}+\lambda s_{23} c_{13}+c_{23} c_{13} A \lambda^{3}(\rho+i \eta)\right| \\
& \quad=\mid A \lambda^{3}(1-\rho+i \eta) c_{13} c_{12} \\
& \quad+A \lambda^{2}\left(s_{12} c_{23}+c_{12} s_{23} s_{13} e^{i \delta}\right)+s_{12} s_{23}-c_{12} c_{23} s_{13} e^{i \delta} \mid .
\end{aligned}
$$

Note that (16) cannot be satisfied when $\theta_{13}=0$. Keeping in mind that $\sin \theta_{13}<\lambda$, the $\lambda^{2} s_{13}^{2}$ and $\lambda^{3} s_{13}$ terms can be neglected since they are of the same order of magnitude as the terms dropped in the Wolfenstein parametrization. Then, we get a simple expression for the cosine of the Dirac CP phase:

$\cos \delta$

$$
\begin{aligned}
= & \frac{s_{12}^{2} s_{23}^{2}+c_{12}^{2} c_{23}^{2} s_{13}^{2}-s_{13}^{2}-\lambda^{2} B}{2 s_{23} c_{23} s_{12} c_{12} s_{13}+2 \lambda s_{23} c_{13} s_{13}+2 A \lambda^{2} s_{12} c_{12}\left(c_{23}^{2}-s_{23}^{2}\right) s_{13}} \\
& +\mathcal{O}\left(\lambda^{4}\right),
\end{aligned}
$$

where $B=s_{23}^{2} c_{13}^{2}-2 A s_{12}^{2} c_{23} s_{23}-2 A \lambda(1-\rho) c_{12} s_{12} c_{13} s_{23}$. We see that for very small $\theta_{13}$ the numerator of the above equation is always larger than the denominator, so that there is no solution for $\delta$.

Using the currently favored CKM [21] and PMNS [5] parameters with their respective uncertainties and solving the condition $\left|U_{13}\right|=\left|U_{31}\right|$ numerically without any approximation, we find that the Dirac CP phase $\delta$ in the PMNS matrix lies between $-32.4^{\circ}$ and $32.0^{\circ}$ at $2 \sigma$. The asymmetry around 0 is due to the small CP violation in the CKM matrix, which does not enter the approximate result in (17).
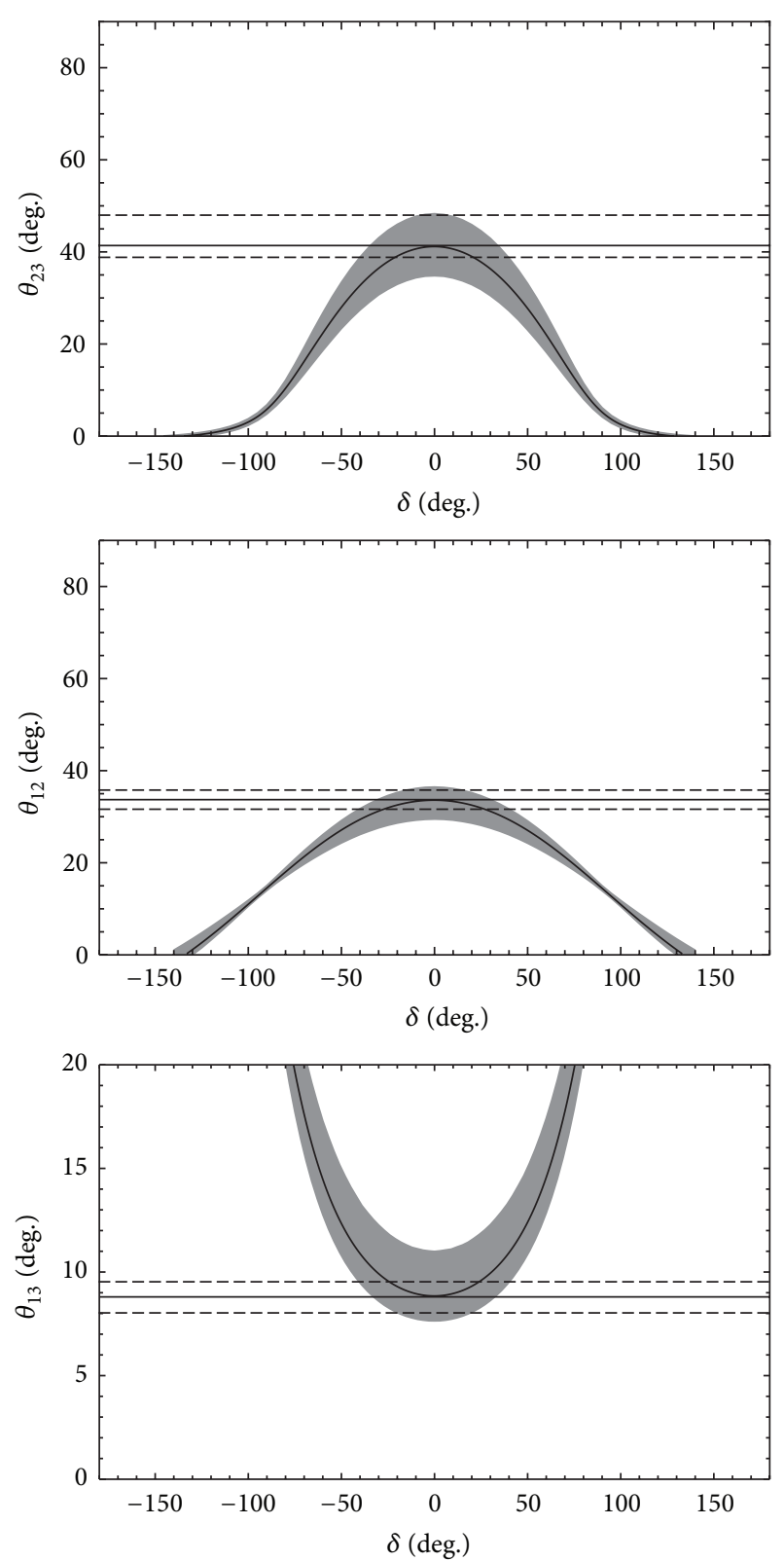

FIGURE 1: The $2 \sigma$ allowed regions (shaded bands) in the $\left(\delta, \theta_{13}\right),(\delta$, $\left.\theta_{12}\right)$, and $\left(\delta, \theta_{23}\right)$ planes using measurements (with uncertainties) of the other two neutrino mixing angles and the CKM parameters. The solid curves within the shaded bands are the model predictions for the best-fit values of the other two mixing angles and the CKM parameters. The horizontal solid lines mark the best-fit values and the horizontal dashed lines mark the $2 \sigma$ limits of $\theta_{23}, \theta_{12}$, and $\theta_{13}$.

We also find predictions for each mixing angle versus $\delta$ given the best-fit values and $2 \sigma$ allowed regions of the other two mixing angles and the CKM parameters. The results are shown in Figure 1. With the constraints from the other two mixing angles and the CKM parameters, we find that $\theta_{23}<48.3^{\circ}, \theta_{12}<36.3^{\circ}$ and $\theta_{13}>7.64^{\circ}$ at $2 \sigma$. The partial QLU model is perfectly consistent with the current data, and rather large $\theta_{13}$ is strongly favored for the measured solar and 
atmospheric mixing angles. Note that the relevant neutrino mass squared differences are trivially accommodated.

A measurement of $\delta$ by future long baseline neutrino oscillation experiments will provide a stringent test of the viability of the partial quark-lepton universality model.

\section{Conclusion}

We studied partial quark-lepton universality, which can naturally arise in grand unified theories. Constraints on the model can be reduced to one simple condition, $\left|U_{13}\right|=\left|U_{31}\right|$. Dropping terms of order $\lambda^{4}$ from this condition, we find a simple expression for the Dirac CP phase $\delta$ in the neutrino sector. We also studied the allowed parameter regions of the model numerically. Our prediction that $\delta$ lies within the range $\left[-32.4^{\circ}, 32.0^{\circ}\right]$ at the $2 \sigma$ level will be tested by future long baseline neutrino experiments.

\section{Conflict of Interests}

The authors declare that there is no conflict of interests regarding the publication of this paper.

\section{Acknowledgment}

This research was supported by the U.S. Department of Energy Grant no. DE-SC0010504.

\section{Endnotes}

1. Implementing a Hermitian $M_{d}$ in a GUT is difficult because $\mathrm{SU}(5)$ does not incorporate left-right symmetry, and, in $\mathrm{SO}(10)$, the mass matrices arising from the couplings of fermions to Higgs fields in the 10 and 126 representations are complex symmetric (and not Hermitian), while those arising from couplings to 120 are complex antisymmetric.

2. An example in which (3), (4), (7), and the Hermiticity of $M_{d}$ naturally arise is an $\mathrm{SO}(10)$ scheme with the superpotential terms [6]

$$
\begin{aligned}
W_{d}= & \frac{f_{i j}}{M}\left(16_{i}^{T} B \Gamma_{\mu} H\right)\left(H^{\prime T} B \Gamma_{\mu} 16_{j}\right) \\
& +\frac{f_{i j}^{\prime}}{M}\left(16_{i}^{T} B \Gamma_{\mu} H^{\prime}\right)\left(H^{T} B \Gamma_{\mu} 16_{j}\right), \\
W_{u}= & g_{i j}\left(16_{i}^{T} B \Gamma_{\mu \nu \lambda \sigma \rho} 16_{j}\right) \Phi^{\mu \nu \lambda \sigma \rho},
\end{aligned}
$$

where $H, H^{\prime}$ are 16-plet Higgs, $\Phi$ is a $\overline{126}$-plet Higgs, $B$ is a charge conjugation matrix in $\mathrm{SO}(10), i$ and $j$ are generation indices, and $\mu, \nu, \lambda, \sigma$, and $\rho$ are $\mathrm{SO}(10)$ indices. The Lorentz indices and the standard charge conjugation matrix are suppressed. $H$ and $H^{\prime}$ contain neutral fields with the quantum numbers of $v$ and $v^{c}$, so that the vacuum expectation value for $v^{c}$ breaks $\mathrm{SO}(10)$ while $\mathrm{SU}(5)$ is preserved. We take the $\overline{126}$ contribution to $H_{d}$ to be zero or subdominant compared to $H$ and
$H^{\prime}$, so $M_{d}$ is only generated from $W_{d}$. By imposing an additional symmetry, $16 \rightarrow 16^{*}, H \rightarrow H^{\prime *}$, which leads to $f_{i j} \rightarrow f_{i j}^{\prime *}$, a Hermitian $M_{d}$ can be obtained.

\section{References}

[1] F. P. An, J. Z. Bai, A. B. Balantekin et al., "Observation of electron-antineutrino disappearance at Daya Bay," Physical Review Letters, vol. 108, no. 17, Article ID 171803, 7 pages, 2012.

[2] F. P. An, Q. An, J. Z. Bai et al., "Improved measurement of electron antineutrino disappearance at Daya Bay," Chinese Physics C, vol. 37, no. 1, Article ID 011001, 2013.

[3] J. K. Ahn, S. Chebotaryov, J. H. Choi et al., "Observation of reactor electron antineutrinos disappearance in the RENO experiment," Physical Review Letters, vol. 108, no. 19, Article ID 191802, 6 pages, 2012.

[4] Y. Abe, C. Aberle, J. C. dos Anjos et al., "First measurement of $\theta 13$ from delayed neutron capture on hydrogen in the Double Chooz experiment," Physics Letters B, vol. 723, no. 1-3, pp. 6670, 2013.

[5] F. Capozzi, G. L. Fogli, E. Lisi, A. Marrone, D. Montanino, and A. Palazzo, "Status of three-neutrino oscillation parameters, circa 2013," Physical Review D, vol. 89, no. 9, Article ID 093018, 2014.

[6] A. S. Joshipura and A. Y. Smirnov, "Quark-lepton universality and large leptonic mixing," Nuclear Physics B, vol. 750, no. 1-2, pp. 28-44, 2006.

[7] K. A. Hochmuth and W. Rodejohann, "On symmetric lepton mixing matrices," Physics Letters B, vol. 644, pp. 147-152, 2007.

[8] Y. BenTov and A. Zee, "The neutrino mixing matrix could (almost) be diagonal with entries \pm 1 ," Physics Letters B, vol. 714, no. 1, pp. 80-84, 2012.

[9] W.-Z. Guo and M. Li, "A possible symmetric neutrino mixing ansatz," Physics Letters B, vol. 718, no. 4-4, pp. 1385-1389, 2013.

[10] C. H. Albright, K. S. Babu, and S. M. Barr, "Minimality condition and atmospheric neutrino oscillations," Physical Review Letters, vol. 81, no. 6, pp. 1167-1170, 1998.

[11] P. Kielanowski, S. R. Juarez Wysozka, and H. J. G. Mora, "Theorems on the renormalization group evolution of quark Yukawa couplings and CKM matrix," Physics Letters B, vol. 479, no. 1-3, pp. 181-189, 2000.

[12] M. Lindner, M. Ratz, and M. A. Schmidt, "Renormalization group evolution of Dirac neutrino masses," Journal of High Energy Physics, vol. 2005, no. 9, article 081, 2005.

[13] J. A. Casas, J. R. Espinosa, A. Ibarra, and I. Navarro, "General RG equations for physical neutrino parameters and their phenomenological implications," Nuclear Physics B, vol. 573, no. 3, pp. 652-684, 2000.

[14] K. R. S. Balaji, A. S. Dighe, R. N. Mohapatra, and M. K. Parida, "Radiative magnification of neutrino mixings and a natural explanation of the neutrino anomalies," Physics Letters B, vol. 481, no. 1, pp. 33-38, 2000.

[15] N. Haba, Y. Matsui, and N. Okamura, "The effects of Majorana phases in three-generation neutrinos," The European Physical Journal C-Particles and Fields, vol. 17, no. 3, pp. 513-520, 2000.

[16] P. H. Chankowski and S. Pokorski, "Quantum corrections to neutrino masses and mixing angles," International Journal of Modern Physics A, vol. 17, no. 5, pp. 575-614, 2002.

[17] S. Antusch, J. Kersten, M. Lindner, M. Ratz, and M. A. Schmidt, "Running neutrino mass parameters in see-saw scenarios," Journal of High Energy Physics, vol. 2005, no. 3, article 024, 2005. 
[18] M. A. Schmidt and A. Y. Smirnov, "Quark lepton complementarity and renormalization group effects," Physical Review D, vol. 74, Article ID 113003, 2006.

[19] G. C. Branco and P. A. Parada, "Is the Cabibbo-KobayashiMaskawa matrix symmetric?” Physical Review D, vol. 44, no. 3, pp. 923-926, 1991.

[20] L. Wolfenstein, "Parametrization of the kobayashi-maskawa matrix," Physical Review Letters, vol. 51, no. 21, pp. 1945-1947, 1983.

[21] M. Bona, M. Ciuchini, E. Franco et al., "The 2004 UTfit collaboration report on the status of the unitarity triangle in the standard model," Journal of High Energy Physics, vol. 2005, no. 7, article 028, 2005, http://www.utfit.org/. 

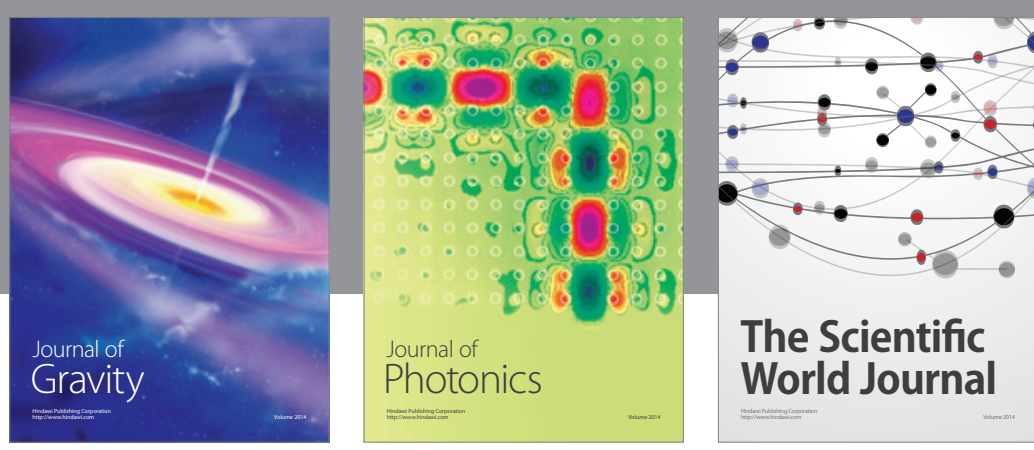

The Scientific World Journal
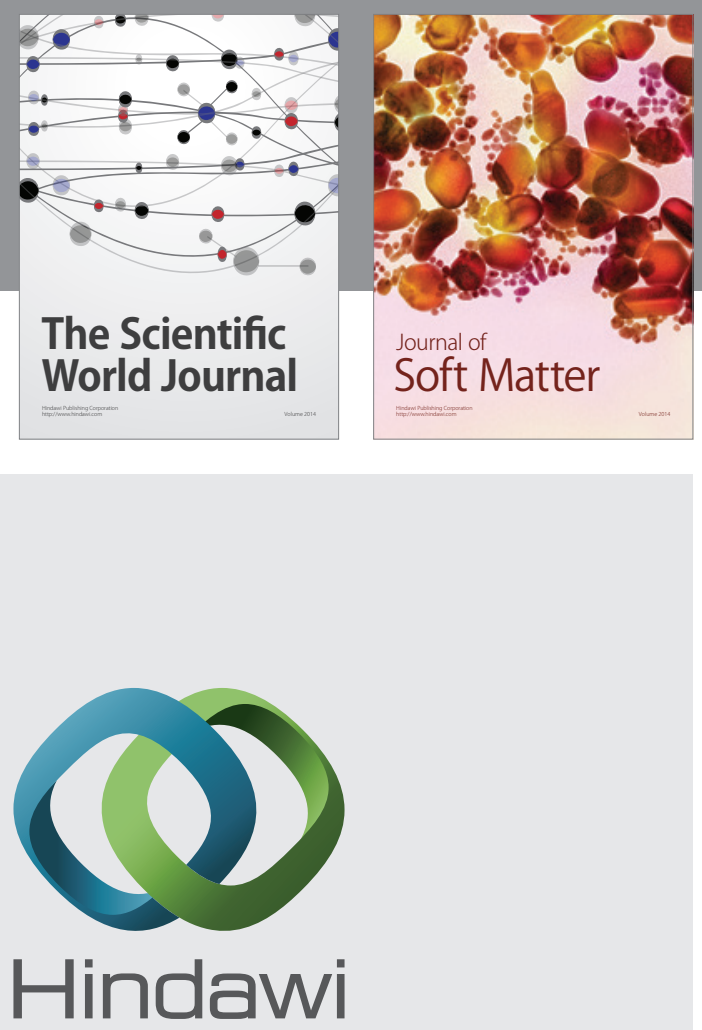

Submit your manuscripts at

http://www.hindawi.com

nternational Journal of

Statistical Mechanics
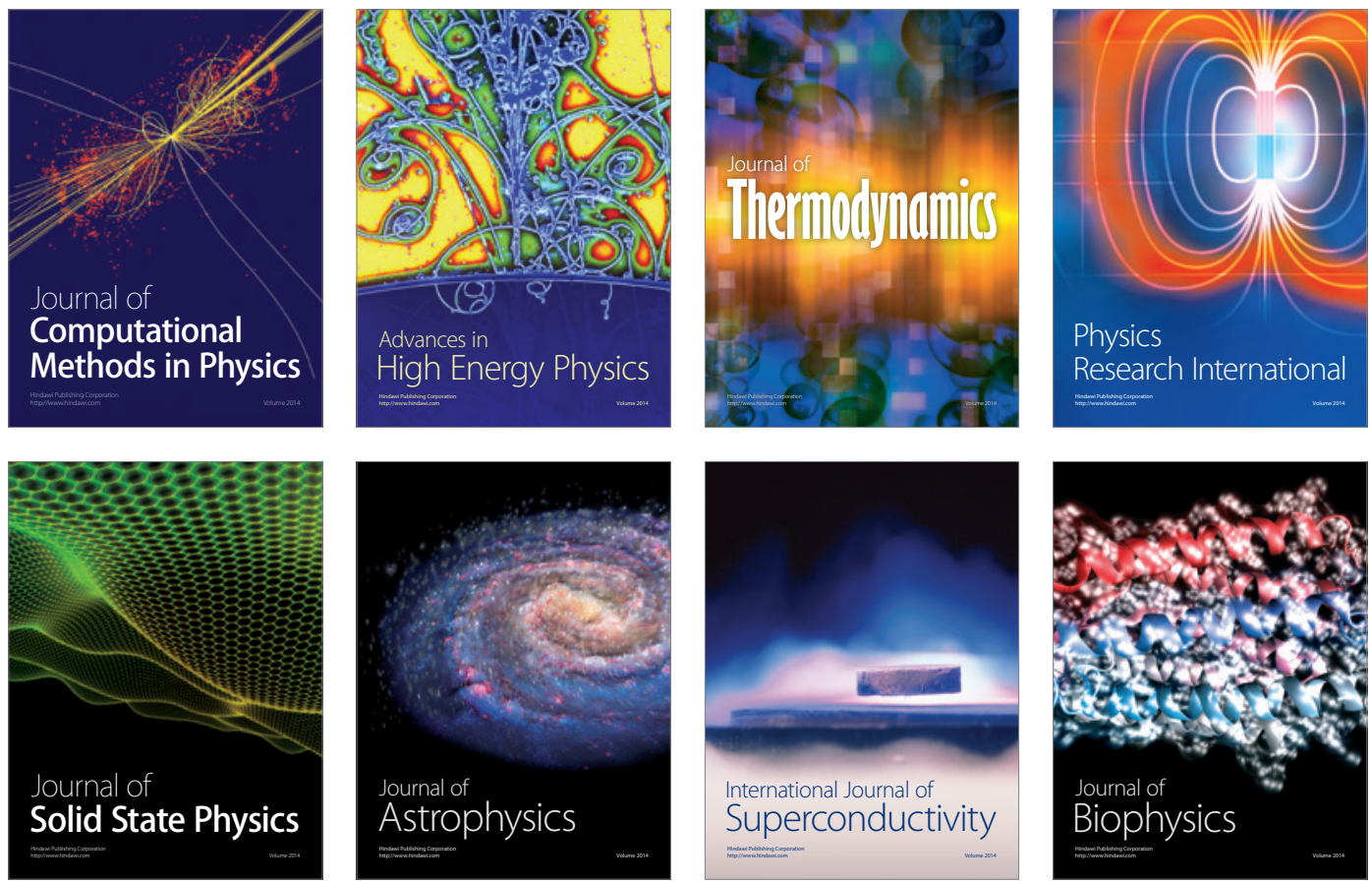
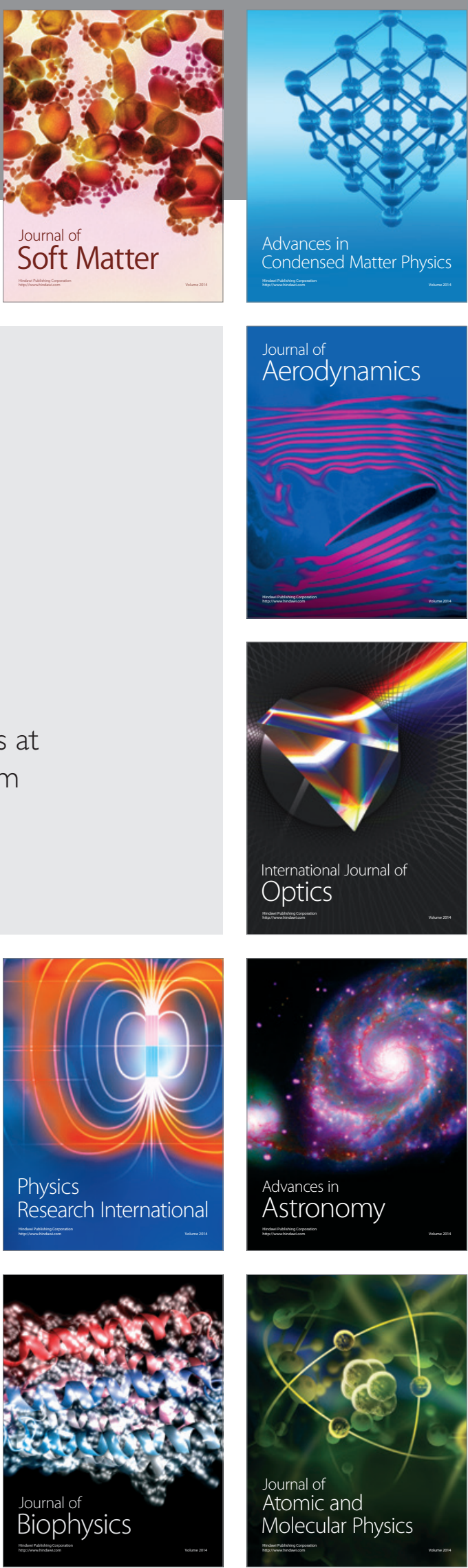